\title{
Macro and nano scale modelling of water-water interactions at ambient and low temperature: relaxation and residence times.
}

María Carmen Morón ${ }^{\text {a,b, }}$, Diego Prada-Gracia ${ }^{c}$ and Fernando Falo ${ }^{\text {b,d }}$

a Instituto de Ciencia de Materiales de Aragón (ICMA), Consejo Superior de Investigaciones Científicas-Universidad de Zaragoza, Pedro Cerbuna 12, E-50009 Zaragoza, Spain. E-Mail: nina@unizar.es.

b Departamento de Física de la Materia Condensada, Facultad de Ciencias, Universidad de Zaragoza, Zaragoza, Spain.

c Freiburg Institute for Advanced Studies, School of Soft Matter Research, Albertstrasse 19, 79104 Freiburg im Breisgau, Germany.

${ }^{d}$ Instituto de Biocomputación y Física de Sistemas Complejos (BIFI), Universidad de Zaragoza, Zaragoza, Spain.

\footnotetext{
* Corresponding author:

María-Carmen Morón

Instituto de Ciencia de Materiales de Aragón

Universidad de Zaragoza

Pedro Cerbuna 12

E-50009 Zaragoza

Spain

E-mail:nina@unizar.es

E-mail: oenola@gmail.com
} 


\begin{abstract}
The decay dynamics of ambient and low temperature liquid water has been investigated through all-atom molecular dynamics simulations, residence times calculations and time correlation functions from $300 \mathrm{~K}$ down to $243 \mathrm{~K}$. Those simulations replicate the experimental value of the self-diffusion constant as a function of temperature by tuning the damping factor of the Langevin equation of motion. A stretched exponential function $\exp \left[-(\mathrm{t} / \tau)^{\beta}\right.$ ] has been found to properly describe the relaxation of residence times calculated at different temperatures for solvent molecules in a nanodrop of free water modelled as a sphere of nanometric dimensions. As the temperature goes down the decay time $\tau$ increases showing a divergence at $T_{s}=227 \pm 3$ $\mathrm{K}$. The temperature independence of the dimensionless stretched exponent $\beta=0.59 \pm$ 0.01 suggests the presence of, not a characteristic relaxation time (since $\beta \neq 1$ ), but a distribution of decay times that also holds at low temperature. An explanation for such heterogeneity can be found at the nanoscopic level. Moreover it can be concluded that the distribution of times already reported for the dynamics of water surrounding proteins ( $\beta \leq 0.5$ ) can not be exclusively due to the presence of the biomolecule itself since isolated water also exhibits such behaviour. The above reported $\mathrm{T}_{\mathrm{s}}$ and $\beta$ values quantitatively reproduce experimental data.
\end{abstract}




\section{Introduction}

Water affects every aspect of our lives. Being involved in nearly all processes it is the most common molecular substance. Although water is a very small molecule, its peculiar properties have a key role in many important physical, biological, chemical, and technical processes. Many of those unusual properties are attributable to the ability of its molecules to form hydrogen bonds with other water molecules forming extended three-dimensional networks. As a result, the dynamics of water will be dominated by the behaviour of the three-dimensional hydrogen-bonded network, parts of which are continuously rebuilt. ${ }^{1-2}$

Despite the relevance of liquid water in nature, many physical properties of this solvent are far from being fully understood. Macroscopic bulk water can remain liquid below its melting point down to approximately 232 - $235 \mathrm{~K}^{3-8}$ staying in a metastable phase ${ }^{9}$. This is possible because in the absence of a nuclei to induce crystallization that universal solvent can be cooled below its freezing temperature without crystallizing since the liquid-solid transition is first order. ${ }^{10}$ Experimental evidence has been also found for the existence of metastable bulk liquid water down to temperatures of $227 \mathrm{~K}$ in micrometre-sized droplets. ${ }^{7,11-12}$. The dynamics of liquid water below $273.15 \mathrm{~K}$ attracts considerable interest since this particular type of water is present in nature in clouds and exhibits a relevant role in meteorological and atmospheric phenomena. ${ }^{13-15}$ This special liquid is also of interest due to its technological importance in diverse areas as aviation ${ }^{10}$, commercial freeze-drying and lyophilisation ${ }^{16}$, cryopreservation $^{17}$ or pharmaceutics $^{18}$.

Several thermodynamic (e.g. isothermal compressibility, thermal expansion coefficient, isobaric heat capacity) and dynamic (e.g. diffusivity, viscosity, structural relaxation) magnitudes of liquid water show an anomalous behaviour. ${ }^{19}$ As the temperature decreases from $273.15 \mathrm{~K}$ the experimental values of these physical observables clearly diverge, with an extrapolated singular temperature $\mathrm{T}_{\mathrm{S}}$ of $228 \mathrm{~K}$ at ambient pressure. ${ }^{20}$ This unexpected first observation has led to an intense curiosity and has promoted numerous experimental and theoretical studies. Both, experiments and computer simulations suggest that the singularity is a purely dynamical transition. ${ }^{21-22}$ However despite the wide interest and the numerous research efforts on this subject, the nature of the phenomena remain unclear and lively debated. ${ }^{4,23-26}$

Experiments in the proximities of $\mathrm{T}_{\mathrm{S}}$ are extremely difficult due to ice formation processes. ${ }^{27}$ Since, as stated above, the singular temperature has been estimated to occur at $\mathrm{T}_{\mathrm{S}} \approx 228 \mathrm{~K}$, the liquid must survive in its metastable state long enough to equilibrate, and the experimental observation time need to be smaller than the nucleation time. Moreover small sample techniques are required to perform measurements under conditions of metastability. In principle those requirements are easier to meet in computer simulations of free water. The details of processes occurring at molecular length at different time scales can be studied using molecular dynamics simulations. Those analyses provide information that is often difficult to obtain experimentally. In this aspect the decay dynamics of liquid water has received significant attention from that particular scientific community. Thus magnitudes as the self intermediate scattering function, which can be measured by neutron scattering experiments, have been calculated. ${ }^{28-30}$ Molecular dynamics simulations of the Kerr effect ${ }^{31}$ or hydrogen bond dynamics $^{32}$ have been also performed to obtain decay data from time correlation functions. However all those computer approximations fail to quantitatively reproduce the experimental value $\mathrm{T}_{\mathrm{s}} \approx 228 \mathrm{~K}$ mentioned above. 
On the other hand the water molecules located within the solvation shell in the immediate vicinity of a biomolecule were termed as 'biological water' by Nandi and Bagchi. ${ }^{33}$ Two kind of water can be differentiated: bound and free. Bound water is attached to the biomolecule (charged or polar amino acid groups) by strong hydrogen bonds. Free water defines solvent molecules that exchange with bound water. Beyond that special solvation shell molecules would behave as regular water. Biological water often exhibits longer lifetimes than the bulk. ${ }^{33-40}$ If macroscopic data are considered (i.e. relaxation times), the dynamics of biological water is affected only to a small extent (24-fold slowdown) when compared with neat water. ${ }^{41-42}$ However if microscopic data are taken into account, that hydration water dynamics exhibits a slow component neatly smaller than that of bulk water (2-3 orders of magnitude). ${ }^{38,43}$ The residence times of water molecules located in the first hydration shell of commonly studied proteins have been recently shown to exhibit multiple time scales with a significant temporal disorder in the system. ${ }^{43-45}$ The question is if that particular dynamics of biological water is solely due to the presence of the protein or, by the contrary, the dynamics of residence times in free water would also exhibit such heterogeneity of relaxation processes.

The paper is organized as follows. Section 2 presents the details of the all-atom molecular dynamics simulations carried out for free water at ambient and low temperature down to $243 \mathrm{~K}$. We have performed those simulations taking into account that the self-diffusion constant of the solvent molecules decreases with temperature. In Section 3 the presence of multiple time scales in water is analysed through time correlation functions (macroscopic view) and residence times (nanoscopic view) at different temperatures. Those results are discussed in Section 4 and compared with previously reported experimental and simulated data as well as theoretical models. The main conclusions are summarised in Section 5.

\section{Computational details}

Classical all-atom molecular dynamics simulations were conducted at $300 \mathrm{~K}$ on free water using a double precision version of the GROMACS software package. ${ }^{46-47}$ The simulated cubic box of $6.2 \times 6.2 \times 6.2 \mathrm{~nm}^{3}$, with periodic boundary condition to prevent boundary effects, contains 7918 water molecules. The simulations were conducted integrating the equations of motion employing Langevin dynamics with a time step (resolution) of 2 fs (0.002 ps). The cutoff length for the Lenard-Jones and Coulomb potentials was set at $1.4 \mathrm{~nm}$. The three site simple point charge model (SPC) was used for modelling water. ${ }^{48}$ In order to follow the dynamics of the solvent at that temperature, a trajectory of 10 nanoseconds was simulated. It was performed in the canonical ensemble, i.e. at constant $\mathrm{N}, \mathrm{V}$ and $\mathrm{T}$. In order to reach a correct stabilization of the simulated system, we waited 3 ns before starting to collect data. The production run, which amounts $7 \mathrm{~ns}$, was saved every 0.1 ps for later analysis.

With the aim of capturing the dynamics of liquid water at low temperature, equivalent simulations were performed at various temperatures below $300 \mathrm{~K}$ down to $243 \mathrm{~K}$. The length of the trajectories was increased at the lower temperatures. As a result, the dynamics of the total 7918 water molecules was monitored during 10 ns for simulations at $288 \mathrm{~K}$ and $273 \mathrm{~K}, 16 \mathrm{~ns}$ at $258 \mathrm{~K}, 25 \mathrm{~ns}$ at $248 \mathrm{~K}$ and $30 \mathrm{~ns}$ at $243 \mathrm{~K}$. As stated above, in order to reach a correct stabilization of the simulated systems, we waited various nanoseconds before starting to collect data: as an example, 3 ns at $288 \mathrm{~K}$ but $18 \mathrm{~ns}$ at $243 \mathrm{~K}$. Finally, the production runs amount $7 \mathrm{~ns}$ for $288 \mathrm{~K}$ and $273 \mathrm{~K}$, but 9 ns, 10 ns or 12 ns for, respectively, 258, 248 and $243 \mathrm{~K}$. The root mean square deviation for all the temperatures considered in this work is $1 \mathrm{~K}$. 
Since the present work refers to the dynamics of residence times calculated for water molecules as temperature is decreased, the above simulations should replicate, as close as possible, the experimental value of the self-diffusion constant $\left(D_{\exp }\right)$ at each temperature. The replication has been done tuning the coupling constant time taut in the damping factor $\gamma=m /$ taut of the Langevin equation of motion $m\left(\mathrm{~d}^{2} \mathrm{r} / \mathrm{dt}^{2}\right)=\mathrm{F}-\gamma(\mathrm{dr} / \mathrm{dt})+$ $\eta(\mathrm{t})$, where $m, \mathrm{r}$ and $\mathrm{F}$ are, respectively, the mass, the position and the force field acting on the atom under consideration in the dynamics. $\eta(t)$ is a noise term with correlation function $\left\langle\eta_{\mathrm{i}}(\mathrm{t}) . \eta_{\mathrm{j}}\left(\mathrm{t}^{\prime}\right)>=2 \mathrm{k}_{\mathrm{B}} \mathrm{T}(\mathrm{m} /\right.$ taut $) \delta_{\mathrm{i}, \mathrm{j}} \delta\left(\mathrm{t}-\mathrm{t}^{\prime}\right){ }^{46-47}$ The quality in the reproduction of $D_{\text {exp }}$ is shown by obtaining the corresponding calculated self-diffusion constant $D_{\text {calc. }}$. The values of taut together with $D_{\text {exp }}$ and $D_{\text {calc }}$ are shown in Table 1 as a function of the temperature.

\section{Results}

The dynamics of ambient and low temperature water is analysed in this section through residence times and correlation functions also including a nanoscopic approximation to the problem.

\subsection{Relaxation at room temperature}

The dynamics of water can be described by evaluating, as a function of time, the occupation of the water molecules on a given spatial arrangement (i.e. a spherical region). That occupancy is related to the mobility (diffusion coefficient) of the water molecules through the region considered. Indeed, the dynamical properties of a fluid can be obtained from time correlation functions. ${ }^{49-53}$ Thus we have considered a normalized occupation function defined as

$$
C(t)=\sum_{i=1}^{N} \frac{\left\langle O_{i}\left(t_{0}\right) O_{i}\left(t_{0}+t\right)\right\rangle}{\left\langle O_{i}\left(t_{0}\right) O_{i}\left(t_{0}\right)\right\rangle}
$$

where $O_{i}\left(t_{0}+t\right)$ takes the value of 1 (unity) if the water molecule $i$, which is within the region of interest at the time origin $t_{o}$, still exists in that region at time $t_{o}+t$. Otherwise $O_{i}\left(t_{o}+t\right)$ equals $0 . \quad \mathrm{N}$ is the total number of water molecules within the region considered at $t_{0}$. The angular brackets denote averaging over various time origins with the aim of improving statistics as well as taking into account different areas of the trajectory. Therefore $C(t)$ gives the average number of water molecules that still remain in the region of interest after a time $t$. In our case such a region is a nanodrop of free water that has been modelled as a sphere with a radius of $2.0 \mathrm{~nm}$ in order to improve statistics. This sphere has been centred in the simulation box. The choice of that cutoff is adequate because we are also interested in comparing the dynamics of water in the first hydration shell of proteins with that of free water. A radius of $2.0 \mathrm{~nm}$ approximates half the diameter of some small proteins of interest plus its first hydration shell. ${ }^{43,54}$

Thus, the function $C(t)$ was calculated following equation (1) with a resolution of 0.1 ps, using the data obtained from all-atom molecular dynamics simulations at $300 \mathrm{~K}$ (Fig. 1, inset). The tendency of the curve consists of a fast initial decay followed by a slower one. This trend cannot be described using a single exponential law. In fact the referred relaxation curve $C(t)$ can be accurately modelled using a stretched exponential function $\exp \left[-(\mathrm{t} / \tau)^{\beta}\right.$ ] (see Fig. 1, inset). ${ }^{55-56}$ This function, which contains just two free parameters: the effective relaxation time $\tau$ and the stretching exponent $\beta$ ( $0<\beta \leq$ 1 ), was proposed empirically more than 150 years ago for its accuracy and simplicity in describing decay data. The stretched exponential, also known as the KWW (KohlraushWilliams-Watts) function, is often observed in several phenomena in complex 
condensed matter systems and supercooled liquids. ${ }^{57}$ The fit of our data to that expression supplies parameter values $\tau \square 312 \pm 3$ ps and $\beta=0.58 \pm 0.01$. As expected, the relaxation time $\tau$ decreases with the radius $r$ of the sphere considered down to, for example, $75.3 \pm 0.5$ ps for $r=1.0 \mathrm{~nm}$.

The time constant $\tau$ gives an estimation of the permanence time of water molecules in the solvent region under study, providing the overall time scale over which the process develops. The exponent of the KWW function also provides interesting information as for example how large is the deviation of the relaxation curve from a classical exponential since for $\beta=1$ the mathematical expression of a simple exponential is restored. In our case, the large stretching effect $(\beta \approx 0.6)$ indicates a considerable large deviation from a classical exponential decay showing that water dynamics is characterized by, not a unique relaxation time (since $\beta \neq 1$ ), but a distribution of different time scales. That finding suggests the presence of significant temporal disorder in the system. ${ }^{21,57}$ Stretched exponentials are frequently employed for modelling phenomena characterized by multiple relaxation rates. ${ }^{58}$ As an example KWW exponents different from 1 were described for the hydration water of various proteins $^{43-45,59}$ and for diffusion-controlled processes in molecular luminescence ${ }^{60}$. Therefore the heterogeneity of decay times reported for the dynamics of water located in the first hydration shell of those proteins, with a stretched exponent of $\beta \leq 0.5$, is not exclusively due to the presence of the biomolecule itself since isolated water also exhibits a distribution of different relaxation times as shown by the large departure from $\beta=1$ it exhibits ( $\beta=0.58 \pm 0.01$ ). A particular case is that of water clusters partially or largely confined inside proteins and in the interior of reverse micelles. For that special kind of solvent molecules the dynamics of water-biomolecule hydrogen bonds has been reported to be slower than that of hydration water. ${ }^{61-63}$ Generally clusters of water confined in proteins exhibit even smaller $\beta$ values than those reported for hydration water, ${ }^{61-62}$ indicating a more heterogeneous hydrogen bonding of those particular kind of solvent with the biomolecule, when compared with hydration and bulk water.

Moreover we have also calculated the value of the $\beta$ parameter for spheres of bulk water with a radius of 1.5 and $1.0 \mathrm{~nm}$. A departure from $\beta=1$ is always found with a constant value $\beta=0.58 \pm 0.01$. The fact that $\beta$ is not affected by the radius of the sphere confirms the independence of the temporal disorder of the system versus the dimensions of that sphere. That finding lets to calculate $\beta$ independently of the radius in consideration. We will exploit this useful result in the following.

\subsection{Relaxation at low temperature}

What is going to happen with those findings if the temperature is decreased from room temperature? In a macroscopic bulk sample several experimental difficulties limit the minimum reachable temperature for liquid water at about an effective $243 \mathrm{~K}^{23}$ As reported in the introduction section, the dynamics of liquid water in this temperature regime is of considerable importance due to its technological relevance (atmospheric phenomena $^{13}$, cryopreservation $^{17}$, commercial freeze-drying and lyophilisation ${ }^{16}$, aviation $^{6}$, pharmaceutics ${ }^{18}$ etc). With the aim of answering the above question, we have carried out molecular dynamics simulations for free water below $300 \mathrm{~K}$ down to $243 \mathrm{~K}$.

The oxygen-oxygen radial distribution functions, $g_{\mathrm{oo}}(\mathrm{r})$, have been calculated for pure water at the above temperatures (see Fig. 2). These atom-atom pair correlation functions are one of the principal structural descriptors for water. As expected, the three characteristic peaks of liquid water appear as the temperature goes down, in agreement with both experimental and simulated results. ${ }^{22,64-65}$ The sharpness of the peaks with the temperature reduction illustrates the progressive ordering of the liquid. 
Concerning relaxation curves calculated from time correlation functions, we do not report here the stretched parameters corresponding to a sphere of radius $2.0 \mathrm{~nm}$ due to the rapid increase of the relaxation time $\tau \square$ as the temperature decreases. Much longer simulation times would be required in order to obtain reliable stretched parameters. Thus below room temperature, and taking advantage of the independence of $\beta$ with the radius of the sphere considered (see Section 3.1), we have calculated the stretched exponent for a radius equal to $1.0 \mathrm{~nm}$. If the radius of the sphere is decreased the decay time $\tau$ goes down, as expected, and the calculation of reliable enough stretched parameters is possible. Thus for $r=1.0 \mathrm{~nm}$, the fit of the autocorrelation functions (eqn (1) ) to a KWW exponential gives parameters $\tau$ and $\beta$ which are shown in Table 2 for various temperatures (as an example see Fig. 1, for the fit at $243 \mathrm{~K}$ ). The relaxation time $\tau$ increases with temperature reduction, as already reported for the temperature dependence of the relaxation times obtained for the hydrogen bond dynamics of bulk water $^{8}$. A particular behaviour arises from the stretched exponent since the value of that parameter nicely keeps constant from 300 to $243 \mathrm{~K}(\beta=0.59 \pm 0.01)$ indicating an independence of the KWW exponent $\beta$ with temperature (see Table 2 and Fig. 3).

The evolution of the effective decay time $\tau$ with the temperature is shown in Fig. 4 (inset). It was first noted by Speedy and Angell ${ }^{20}$ that many dynamic magnitudes of water (such a isothermal compressibility, viscosity or proton and oxygen spin-lattice relaxation times) change with temperature following a power-law divergence proportional to negative powers of $\left(\mathrm{T}-\mathrm{T}_{\mathrm{s}}\right)$. By extrapolation at low temperatures, those dynamic magnitudes of water would become infinitely large at the 'singular temperature' $\mathrm{T}_{\mathrm{s}}$ with an extrapolated experimental value of $228 \mathrm{~K}$. In order to check if that is the case for the relaxation times $\tau$ of Table 2, their temperature evolution has been fitted to the expression $\tau \sim\left(T-T_{s}\right)^{-\gamma}$. The result is showed in Fig. 4 where a nice linear dependence is found for the evolution of $\tau^{-(1 / \gamma)}$ with temperature. The best fit corresponds to $\mathrm{T}_{\mathrm{s}}=227 \pm 3 \mathrm{~K}$ and $\gamma=1.71 \pm 0.1$.

The exponent $\gamma=1.71 \pm 0.1$ is in agreement with that one governing the power-law vanishing of the experimental diffusion constant with temperature. Thus the fit to the expression $D_{\exp } \sim\left(\mathrm{T}-\mathrm{T}_{\mathrm{s}}\right)^{\gamma}$ gives the parameters $\mathrm{T}_{\mathrm{s}}=227 \mathrm{~K} \pm 1 \mathrm{~K}$ and $\gamma=1.64 \pm 0.10$ (see Fig. 5) . The experimental singular temperature $T_{s}$ has been reported to exhibit the same value for different magnitudes measured on water ${ }^{20,23,66-68}$, as it happens in our case for $\tau$ and $\mathrm{D}\left(\mathrm{T}_{\mathrm{s}}=227 \pm 3 \mathrm{~K}\right.$ and $\mathrm{T}_{\mathrm{s}}=227 \pm 1 \mathrm{~K}$, respectively). Gallo et al ${ }^{69}$ have also found coincidence in the singular temperature $\mathrm{T}_{\mathrm{s}}$ extracted from both diffusion coefficient and intermediate scattering function calculated from molecular dynamics simulations data. However their reported $T_{s}$ value is more than $30 \mathrm{~K}$ smaller than the Speedy and Angell experimental temperature of $228 \mathrm{~K}^{20}$. Those simulations were performed for water close to the centre of a silica pore with an average diameter of 4 $\mathrm{nm}$.

\subsection{Nanoscopic view at room temperature}

To gain a microscopic understanding of the heterogeneity of decay times reported for bulk water, the time that 7918 solvent molecules spend at a distance smaller than 0.40 $\mathrm{nm}$ from a given solvent molecule, taken as a reference, has been calculated along the whole room temperature simulation (several molecules of reference were considered). The results are shown in Fig. 6. The cutoff of $0.40 \mathrm{~nm}$ is also judged as appropriate to compare with water located at the first hydration shell of a biomolecule. ${ }^{43}$ The atomic level analysis, depicted in Fig. 6, shows a wide distribution of residence times, $\mathrm{rt}(\mathrm{r}=0.40 \mathrm{~nm})$, ranging from hundreds of femtoseconds to tens of picoseconds (see also 
Fig. 7, upper part). Please, keep in mind that although the resolution of the simulation is 2 femtoseconds (time step), that value increases up to 100 femtoseconds ( 0.1 ps) in the case of the collected data. That wide distribution of times at a nanoscopic level explain the temporal heterogeneity already detected by the macroscopic results presented in this work (decay process, Section 3.1), that is the large departure from 1 of the stretched exponent $\beta$.

As in the case of relaxation (Section 3.1), a similar heterogeneity of residence times has also been reported for the first hydration shell of the inhibitor Barstar. ${ }^{43}$ That temporal disorder cannot be solely due to the presence of the protein since, as shown above, bulk water also presents a wide distribution of times itself. However water molecules around proteins like for example Insulin ${ }^{45}$ and Barstar $^{43}$ exhibit residence times in the range of 400-700 ps or even higher. The big difference between the two type of water, biological and bulk, is that such ultra long residence times are not detected for bulk water (see Fig. 6 and 7 upper part). The question is why such remarkable difference? When recently studying the dynamics of hydration water around the protein Insulin, Bagchi and Roy $^{45}$ have revealed that the kinetics of the hydrogen bonds for molecules exhibiting higher residence times are lower than those presenting smaller residence times. Moreover we have found that the water molecules that stay longer periods of time near the inhibitor Barstar present more hydrogen bonds with atoms of the biomolecule than with the rest of the solvent, and vice versa. ${ }^{43}$ It is significant to remember that a water-protein hydrogen bond is stronger than a waterwater one. ${ }^{33,70}$ Evidently in the case of bulk water there is no biomolecule present. Slow hydration water is interesting for several reasons. It retards charge transfer processes, ${ }^{34-}$ ${ }^{35}$ prevents dehydration, ${ }^{34}$ increases friction on the surface involved ${ }^{71}$ etc. Moreover slow confined clusters of water molecules also play a central role in protein function, including allosteric regulation and charge transfer. ${ }^{61-62}$

\subsection{Nanoscopic view at low temperature}

In order to explore if the temporal disorder, reported in Section 3.2, is also observed at low temperature, the time that 7918 solvent molecules spend at a distance smaller than $0.40 \mathrm{~nm}$ from a given water molecule taken as a reference has been also calculated at $243 \mathrm{~K}$ (see Fig. 8). Several molecules of reference were considered. A heterogeneity of residence times is also detected when comparing with the data at room temperature (see Fig. 6). However the dynamics at this atomic level clearly became slower as the temperature is going down. As an example, the difference between the residence times at room and low temperature $(243 \mathrm{~K})$ is one order of magnitude. Thus the longest residence times found at $300 \mathrm{~K}$ amounts some tens of picoseconds while those at $243 \mathrm{~K}$ increase up to some hundreds of picoseconds (see also Fig. 7). Same calculations performed for the intermediated temperatures reported above (300 - $243 \mathrm{~K}$ ) agree with the nanoscopic slowing of the dynamics of bulk water as temperature decreases. On the other hand as temperature goes down the dynamics of bulk water approximates that of water molecules in the vicinity of biomolecules in the sense that, as the temperature decreases the residence times of bulk water increase, thus moving through the values reported for biological water (nanoseconds time scale). ${ }^{43,45}$

At this point a question arises: if a water molecule success in being located at the smallest distance of a solvent molecule of reference, would it stay at that particular position during a longer period of time? In other words would those special water molecules be responsible for the long residence times of tens and hundreds of picoseconds detected for bulk water at room and low temperature respectively (see Figs. 6, 7 and 8)? With the aim of answering this question, the time that 7918 molecules of bulk water spend at the smallest distance of a given solvent molecule of reference has 
been calculated. Several molecules of reference were considered. These results are shown at $\mathrm{T}=243 \mathrm{~K}$ in Fig. 9 where it is possible to observe that, at a given time, if a particular water molecule success in being located at the smallest distance of the solvent molecule of reference, that fact does not necessarily implies that this water molecule is going to stay at such a minimum distance during a long period of time. In fact, a distribution of times can be detected (Fig. 9). The same phenomenon of temporal disorder at atomic level can be observed at room temperature but this time the maximum residence time amounts just some tens of picoseconds while at $\mathrm{T}=243 \mathrm{~K}$ this value increases up to some hundreds of picoseconds.

\section{Discussion}

In the following the results obtained in Section 3 are compared with previous data in the literature and discussed in the frame of different theoretical approximations as the diffusion-trap model ${ }^{57}$, the mode coupling theory ${ }^{72-74}$ or the cage effect ${ }^{75-76}$. Special attention is paid to the singular temperature $T_{\mathrm{s}}$ and the stretched exponent $\beta$ as well as the presence of 'defects' within the hydrogen bond network.

\subsection{Relaxation and singular temperature $\mathbf{T}_{\mathrm{s}}$}

The singular temperature $T_{\mathrm{S}}$, extrapolated from experimental data and predicted for liquid water as it is cooled down, throws values as $228 \mathrm{~K}^{20,66}, 227 \mathrm{~K}^{23,67}, 226 \mathrm{~K}^{68}, 221$ $\mathrm{K} \pm 5^{21}$ or $220 \pm 10 \mathrm{~K}^{77}$. Previous molecular dynamics simulations failed to quantitatively reproduce those experimental in character results, since they supplied $\mathrm{T}_{\mathrm{S}}$ $=198.3 \mathrm{~K}^{31}$ (optical Kerr effect), $\mathrm{T}_{\mathrm{S}}=191 \mathrm{~K}^{28}$ and $202 \mathrm{~K}^{30}$ (intermediate scattering function), $\mathrm{T}_{\mathrm{S}}=197.5 \pm 1 \mathrm{~K}^{32}$ (hydrogen-bond dynamics) or $\mathrm{T}_{\mathrm{s}}=185.3 \mathrm{~K}^{69}$ (diffusion coefficient). From the relaxation times $\tau$ depicted in Table 2, we have calculated a singular temperature $\mathrm{T}_{\mathrm{S}}=227 \pm 3 \mathrm{~K}$, as shown in Section 3.2. This value is in quantitative agreement with the experimental results listed above for $T_{S}$. Please mind that the molecular dynamics simulations this work presents, quantitatively reproduce the experimental self-diffusion constant of liquid water as the temperature is decreased (see Section 2 and Table 1).

The mode coupling theory ${ }^{72-74}$ provides a mathematically well-defined model for describing the slow dynamics in low temperature glass forming simple liquids or in molecular liquids with spherical-symmetric interactions between the molecules. On the basis of that theory, the slowing down of residence times showed in Fig. 4 as the temperature decreases (see inset), could be described as a purely dynamic process. Thus that theory, through a dynamical model, predicts the asymptotic power-law divergence of correlation times and also power-law vanishing of the diffusion constant $D$ as the temperature decreases.

\subsection{Relaxation and stretched exponent $\beta$}

In the following we will refer to the dimensionless stretched parameter $\beta=0.59 \pm$ 0.01 and its constant value throughout the entire temperature range considered (see Table 2 and Fig. 3). These two results are in excellent agreement with that reported from experimental determinations for ambient and low temperature liquid water that provides $\beta=0.6$ with independence of the temperature considered. ${ }^{21,49,77}$ Previously reported molecular dynamics simulations of the intermediate scattering function for the system under consideration, have failed to quantitatively reproduce the experimental value of $\beta$ and their constant evolution with temperature ( $\beta$ moves from 0.7 to 0.95). ${ }^{28,69,78}$ However Skaf and Sonoda, performing molecular dynamics simulations of the optical Kerr effect for ambient and low temperature liquid water, have obtained 
values for the stretched exponent within the range $0.56-0.68 .^{31}$ Interestingly a high degree of reproducibility has been reported for the values of $\beta$ measured by different methods on the same material. ${ }^{57}$ A $\beta$ value of 0.6 has been also revealed for systems as different as metallic and oxide glasses, polymers or polar organic alcohols. ${ }^{57,79}$

A physical origin for the stretched exponent $\beta=0.59 \pm 0.01$ (Section 3.1 and 3.2) can be found in the 'diffusion-trap' model in which $\beta$ has an inherently topological origin. ${ }^{57}$ The model considers a uniform distribution of excitations that diffuse to traps where the excitations are annihilate. The excitations that are closest to the traps disappear first and those that are further away take longer times to reach the traps. It is this kind of memory effect that produces the stretched exponential relaxation. The diffusion-trap model was originally developed to describe not molecular but electronic relaxation in amorphous semiconductors, where excitons diffuse to network defects, but paradoxically it describes the latter much more accurately than the former. That theory predicts $\beta=3 / 5$ for short-range forces but $\beta=3 / 7$ for relaxation when long-range forces are present. Therefore the values of the stretched parameter shown in Table $2, \beta=3 / 5$, would indicate that the relaxation process that takes place in ambient and low temperature liquid water is dominated by short-range molecular decay channels dismissing longer-range interactions.

In the diffusion-trap model the stretched parameter is calculated as $\beta=d_{\text {eff }} /\left(d_{\text {eff }}+2\right)$ where $d_{\text {eff }}$ is the effective dimensionality of the pathways involved in the excitations decay and can be expressed as $d_{\text {eff }}=f \times d$, where $d$ is the dimensionality of the system under study, i.e. $d=3$ for structural glasses, and $f$ is the fraction of channels activated for the particular decay process. ${ }^{57,80}$ Since in our case $\beta=0.59 \pm 0.01$, therefore $d_{\text {eff }}=$ $2.88(2.9 \pm 0.1)$. Thus an effective dimensionality of nearly 3 could be assigned to the relaxation process of residence times calculated for solvent molecules in a nanodrop of water at ambient and low temperatures. Since for water $d=3$ it can be deduced that nearly $100 \%$ of the channels are activated $(f=0.96)$. From those considerations it can be deduced that the independence of $\beta$ with temperature, depicted in Fig. 3, would indicate that the nature of the relaxation process, reflected in $d_{\text {eff }}$, does not change when liquid water is cooled down from ambient to $243 \mathrm{~K}$.

\subsection{Diffusion-trap model, residence times and cage effect in the case of water: hydrogen bond network}

Taking the diffusion-trap model as a point of departure and inspiration a question arises: what kind of entities would act as excitons and traps in the case of water? Of significant interest for water is the network of hydrogen bonds it presents. Ab initio investigations of hydrogen bonding in bulk water detect water molecules with two, three, four, and five hydrogen bonds. ${ }^{81}$ Moreover experimental evidences suggest that the ideal tetrahedral network is not perfect but contains 'defects' such an extra (fifth) molecule in the first coordination shell. ${ }^{82-83}$ The two alone non-bonding pairs of electrons interact not with two, as expected from a perfect tetrahedral coordination, but with three protons from three different molecules. These 'defects' would be caused by an increase/reduction of molecules in that shell with respect to a tetrahedral coordination. Therefore it seems to be reasonable that in the case of water, molecules with a coordination number higher than 4 could act as excitons while solvent molecules with a coordination number lower than 4 could behave as traps.

We should test if our simulations can detect the presence of such defects. Thus we have calculated the number of hydrogen bonds that, as a function of time and at atomic level, a given water molecule taken as a reference can establish with their neighbours at room temperature (several molecules of reference were considered). The results are presented in Fig. 10, upper part, where it is shown that the first hydration shell contains 
different kind of 'defects' as a function of time (up to six hydrogen bonds per water molecule). As probably expected, the extreme configurations are much less visited. Moreover investigations considering water reorientation at room temperature, found that in the vast majority of $\mathrm{H}$-bond breaking events, a new $\mathrm{H}$-bond partner is unavailable and the reorientation of the solvent molecule is unsuccessful; successful reorientation would require this availability. ${ }^{84-87}$

On those basis, a water molecule exhibiting a tetrahedral coordination of hydrogen bonds and surrounded by solvent molecules also possessing such a coordination would be a good candidate to high residence times (see nanoscopic view, Section 3.3) because of its lower mobility due to i) a more tetrahedral first hydration shell and ii) less available H-bond partners. By the contrary a water molecule with a high local deviation from tetrahedrality ('defects') and attached to solvent molecules also exhibiting that situation would be a good candidate to low residence times (see nanoscopic view, Section 3.3) because of its higher mobility due to i) a less tetrahedral first hydration shell and ii) more available H-bond 'defects' from the surrounded solvent molecules.

In addition the increase of the decay time $\tau$ as $\square \square \square$ temperature decreases ( $\mathrm{T} \square \square \square \square$, Fig. 4 inset) could be also interpreted considering the dependence of the 'defects' with temperature together with the cage effect ${ }^{75-76}$, i.e. the confinement of molecules due to the interactions with neighbouring molecules. Considering a tagged particle, if the other liquid particles were fixed, the tagged one could not move very far. It would be confined in a cage formed by its neighbours. It can migrate only through rearrangement of the particles surrounding it. The neighbours can move because their neighbours move and so on. On the other hand the local structure of hydrogen bonds evolves more tetrahedral as the temperature goes down, moving through that of ice. ${ }^{8}$ The structure of the liquid changes: a more perfect tetrahedral hydrogen bond network is approached. At this point we should test if our simulations can detect that particular behaviour. Then we have calculated the number of hydrogen bonds that, as a function of time and at atomic level, a given water molecule taken as a reference can establish with their neighbours at low temperature (several molecules of reference were considered). The results are depicted in Fig. 10 (lower part). As at room temperature, the coordination of the first hydration shell as a function of time, shows up to six hydrogen bonds per water molecule. It has been reported for temperatures from 200 to $450 \mathrm{~K}$, that there is a non-vanishing but negligible probability of having six hydrogen-bonded neighbours. ${ }^{89}$ Please compare the upper and lower parts of Fig. 10 where the decrease of 'defects' is evidenced at atomic level as the temperature moves from ambient to $243 \mathrm{~K}$. Therefore as the number of 'defects' decreases on cooling, a given water molecule will be longer confined in its cage due to a higher number of neighbours presenting tetrahedral, and then more stable, coordination. Since the relaxation time $\tau$ would be related to the lifetime of the cage, larger $\tau$ values would be expected for water as the temperature goes down due to a decrease on the number of 'defects'. Recently Saito et al ${ }^{8}$ have calculated the temperature dependence of tetrahedrality of water molecules and found a growth of the tetrahedral structure with temperature reduction down to temperatures as low as $205 \mathrm{~K}$. They have correlated such a growth with the rapid increase in the simulated specific heat $C_{\mathrm{p}}$ down to $\sim 220 \mathrm{~K}$. 


\section{Main Conclusions}

The relaxation of residence times, calculated for solvent molecules located in a nanodrop of free water modelled as a sphere of nanometric dimensions, has been found to be well described by a stretched exponential function $\exp \left[-(\mathrm{t} / \tau)^{\beta}\right]$ at both ambient and low temperature $(300-243 \mathrm{~K})$. As this magnitude decreases $\tau$ increases showing a divergence at a singular temperature $T_{s}=227 \pm 3 \mathrm{~K}$. The value of the stretched exponent $\beta=0.59 \pm 0.01$ indicates the existence of multiple decay time scales and significant temporal disorder in the system. It also shows: i) that the relaxation process is dominated by short range molecular decay channels dismissing longer range interactions, ii) an effective dimensionality of the pathways involved in the excitations decay $\mathrm{d}_{\text {eff }} \approx 3$, and iii) a fraction of activated channels for the relaxation process $f \approx 1$. The independence of $\beta$ with temperature suggests that this mechanism applies not only at ambient but also at low temperature within the range considered $(300-243 \mathrm{~K})$. The presence of such a distribution of decay times for liquid water is explained on the basis of the existence of multiple residence times at the nanoscopic scale.

Such a particular dynamics is maintained as the temperature decreases. The existence of a distribution of residence times, at both room and low temperatures, has been related with the 'cage effect' and with the presence of local deviations from the tetrahedrality ('defects') in the hydrogen bond network. Within the diffusion-trap model scenario, water molecules exhibiting a number of hydrogen bonds higher than 4 are suggested to act as 'excitons' while if that number is lower than 4 they are regarded as 'traps'. The nanoscopic study performed in this work reveals that the residence times of water molecules, around one of them taken as a reference, reach values up to tens of picoseconds at room temperature. However that value increases one order of magnitude up to hundreds of picoseconds at $243 \mathrm{~K}$.

Thus bulk water cannot be judged as responsible for the long residence times reported for room temperature biological water (nanosecond scale) since the longer permanence times observed for neat water are two order of magnitude smaller (some tens of picoseconds). Moreover the existence of multiple time scales and significant temporal disorder in the dynamics of room temperature water placed in the first hydration shell of proteins $(\beta \leq 0.5)$ can not be solely due to the presence of the biomolecule itself since isolated water also shows a distribution of decay times $(\beta=3 / 5$ $\neq 1$ ) and multiple residence times at the nanometric view. Therefore it is possible to conclude that bulk water exhibits a distribution of relaxation and residence times as biological water does, but keeping in mind that a different range of time scales is applicable for each type of water (up to tens of picoseconds in the first case but up to some nanoseconds in the second one).

Finally it is significant to remark that the values obtained in this work for the singular temperature $T_{\mathrm{s}}$ and the stretched exponent $\beta$ are in quantitative agreement, both of them, with those obtained from different experiments performed in liquid water cooled from 300 to $243 \mathrm{~K}$. Our simulations reproduce the experimental value of the self-diffusion constant as a function of temperature. To do that we have tuned the coupling constant time taut in the damping factor $\gamma=m /$ taut of the Langevin equation of motion $m\left(\mathrm{~d}^{2} \mathrm{r} / \mathrm{dt}^{2}\right)$ $=\mathrm{F}-\gamma(\mathrm{dr} / \mathrm{dt})+\eta(\mathrm{t})$ where $\eta(\mathrm{t})$ is a noise term while $m, \mathrm{r}$ and $\mathrm{F}$ are, respectively, the mass, the position and the force field acting on the atom under consideration in the dynamics (see Section 2 for details). That working-schema could be extended to determine different magnitudes depending on the self-diffusion constant of water but also of other liquids. 


\section{Acknowledgements}

Dr. Pedro J. Martínez but also Dr. Alessio Cardillo and Dr. Oscar Y. Fajardo are fully acknowledged for the management and maintenance of the computer cluster where the simulations were performed. This work was funded by the Spanish MICINN (Ministerio de Ciencia e Innovación) and the FEDER (ERDF-European Regional Development Fund) through Projects No. FIS2014-55867-P and FIS2011-25167. Financial support though Grant No. E19 (Gobierno de Aragón, Spain) to Research Group FENOL is also acknowledged. 


\section{References}

1. M. D. Fayer, Acc. Chem. Res., 2011, 45, 3-14.

2. B. Bagchi, 'Water in biological and chemical processes', Cambridge University Press, Cambridge, U.K., 2013, pp. 1-356.

3. G. Franzese and V. Bianco, Food Biophys., 2013, 8, 153-169.

4. E. B. Moore and V. Molinero, Nature, 2011, 479, 506-508.

5. J. C. Kasper and W. Friess, Eur. J. Pharm. Biopharm., 2011, 78, 248-263.

6. L. Liu, A. Faraone, C. Y. Mou, C. W. Yen and S. H. Chen, J. Phys.: Condens. Matter. 2004, 16, S5403-S5436.

7. F. Caupin, J. Non-Cryst. Solids, 2015, 407, 441-448.

8. S. Saito, I. Ohmine and B. Bagchi, J. Chem. Phys., 2013, 138, 094503.

9. S. Sastry, Nature, 1999, 398, 467-470.

10. V.C. Weiss, M. Rullich and C. Kohler and T. Frauenheim, J. Chem. Phys., 2011, 135, 034701.

11. J. A. Sellberg, C. Huang, T. A. McQueen, N. D. Loh, H. Laksmono et al, Nature, 2014, 510, 381-384.

12. H. Laksmono, T. A. McQueen, J. A. Sellberg, N. D. Loh et al, J. Phys. Chem. Lett., 2015, 6, 2826-2832.

13. B. J. Murray, D. O’Sullivan, J. D. Atkinson and M. E. Webb, Chem. Soc. Rev., 2012, 41, 6519-6554.

14. H. R. Pruppacher and J. D. Klett, Microphysics of clouds and precipitation, Kluwer Academic Publishers, Dordrecht, The Netherlands, 1997, pp 1-945.

15. H. R. Pruppacher, Pure Appl. Geophys., 1967, 68, 186-195.

16. R. Geidobler and G. Winter, Eur. J. Pharm. Biopharm., 2013, 85, 214222.

17. G. J. Morris and E. Acton, Cryobiology, 2013, 66, 85-92.

18. J. A. Searles, J. F. Carpenter, T. W. Randolph, J. Pharm. Sci., 2001, 90, 860-871.

19. P. G. Debenedetti, J. Phys.: Condens. Matter, 2003, 15, R1669-R1726.

20. R. J. Speedy and C. A. Angell, J. Chem. Phys., 1976, 65, 851-858.

21. R. Torre, P. Bartolini and R. Righini, Nature, 2004, 428, 296-299.

22. F. Sciortino, P. Gallo, P. Tartaglia and S. H. Chen, Phys. Rev. E, 1996, 54, 6331-6343.

23. A. Taschin, P. Bartonili, R. Eramo, R. Righini and R. Torre, Nature Commun., 2013, 4, 2401.

24. P. H. Poole, R. K. Bowles, I. Saika-Voivold and F. Sciortino, J. Chem. Phys., 2013, 138, 034505.

25. C. A. Angell and Z. Zhao, Faraday Discuss., 2013, 167, 625-641.

26. C. A. Angell, Science, 2008, 319, 582-587.

27. F. Smallenburg, L. Filion and F. Sciortino, Nature Phys., 2014, 10, 653657.

28. P. Gallo and M. Rovere, J. Chem. Phys., 2012, 137, 164503.

29. A. Magno and P. Gallo, J. Phys. Chem. Lett., 2011, 2, 977-982.

30. F. Sciortino, L. Fabbian, S. H. Chen and P. Tartaglia, Phys. Rev. E, 1997, 56, 5397-5404.

31. M. S. Skaf and M. T. Sonoda, Phys. Rev. Lett., 2005, 94, 137802.

32. F. W. Starr, J. K. Nielsen and H. E. Stanley, Phys. Rev. Lett., 1999, 82, 2294-2297. 
33. N. Nandi and B. Bagchi, J. Phys. Chem. B 1997, 101, 10954-10962.

34. K. Bhattacharyya, Chem. Commun. 2008, 25, 2848-2857.

35. B. Bagchi and B. Jana, Chem. Soc. Rev., 2010, 39,1936-1954.

36. T. M. Raschke, Curr. Opin. Struc. Biol., 2006, 16, 152-159.

37. B. Bagchi, Chem. Rev. 2005, 105, 3197-3219.

38. K. Sahu, S. K. Mondal, S. Ghosh and K. Bhattacharyya, Bull. Chem. Soc. Jpn., 2007, 80, 1033-1043.

39. U. Heugen, G. Schwaab, E. Brundermann, M. Heyden, X. Yu, D. M. Leitner and M. Havenith, Proc. Nat. Acad. Sci. USA, 2006, 103, 12301-12306.

40. S. Ebbinghaus, S. J. Kim, M. Heyden, X. Yu, U. Heugen, M.Gruebele, D. M. Leitner and M. Havenith, Proc. Natl. Acad. Sci. USA, 2007, 104, 20749-20752.

41. K. E. Furse and S.A. Corcelli, J. Phys. Chem. Lett., 2010, 1, 1813-1820.

42. L. Nilsson and B. Halle, Proc. Natl. Acad. Sci. USA, 2005, 102, 13867-13872.

43. M. C. Morón, Phys. Chem. Chem. Phys., 2012, 14, 15393-15399.

44. J. Servantie, C. Atilgan and A. R. Atilgan, J. Chem. Phys., 2010, 133, 085101.

45. K. Bagchi and S. Roy, J. Phys. Chem. B, 2014, 118, 3805-3813.

46. D. Van der Spoel, E. Lindahl, B. Hess, G. Groenhof, A. E. Mark and H. J.

C. Berendsen, J. Comput. Chem., 2005, 26, 1701-1718.

47. H. J. C. Berendsen, D. van der Spoel and R. van Drunen, Comput. Phys. Commun., 1995, 91, 43-56.

48. H. J. C. Berendsen, J. P. M. Postma, W. F. van Gunsteren and J. Hermans, in Intermolecular Forces, ed B. Pullman, Reidel Publishing Company, Dordrecht, The Netherlands, 1991, pp 331-342.

49. A. Taschin, P. Bartolini, A. Marcelli, R. Righini and R. Torre, Faraday Discuss., 2013, 167, 293-308.

50. N. Choudhury, J. Phys. Chem. C, 2007, 111, 2565-2572.

51. F. Sciortino, Chem. Phys., 2000, 258, 307-314.

52. F.W. Starr, S. Harrington, F. Sciortino and H. E. Stanley, Phys. Rev. Lett., 1999, 82, 3629-3632.

53. F. W. Starr, F. Sciortino and H. E. Stanley, Phys. Rev. E, 1999, 60, 67576768.

54. J. Sancho, Cell. Mol. Life Sci., 2006, 63, 855-864.

55. G. Williams and D. C. Watts, Trans. Faraday Soc., 1970, 66, 80-85.

56. R. Kohlrausch, Ann. Phys. (Leipzig), 1847, 148, 353-405.

57. J. C. Phillips, Rep. Prog. Phys., 1996, 59, 1133-1207.

58. V. A. Makarov, B. K. Andrews, P. E. Smith and B. M. Pettit, Biophys. J., 2000, 76, 2966-2974.

59. S. Dellerue, M. C. Bellissent-Funel, Chem. Phys., 2000, 258, 315-325.

60. S. A. Rice, in Comprehensive Chemical Kinetics, ed C. H. Bamford, C. F. H. Tipper and R. G. Compton, Elsevier, Amsterdam, 1985, vol 25.

61. Y. Xu, R. Gnanasekaran and D. M. Leitner, Chem. Phys. Lett., 2013, 564, 78-82.

62. R. Gnanasekaran, Y. Xu and D. M. Leitner, J. Phys. Chem. B, 2010, 114, 1698916996.

63. P. A. Pieniazek, Y. S. Lin, J. Chowdhary, B. M. Ladanyi and J. L. Skinner, J. Phys. Chem. B, 2009, 113, 15017-15028.

64. C. Vega, C. McBride, E. Sanz and J. L. F. Abascal, Phys. Chem. Chem. Phys., 2005, 7, 1450-1456.

65. A. K. Soper, Chem. Phys., 2000, 258, 121-137.

66. C. Ronne, P. O. Astrand and S.R. Keiding, Phys. Rev. Lett., 1999, 82, 
2888-2891.

67. B. D. Cornish and R. J. Speedy, J. Phys. Chem., 1984, 88, 1888-1892.

68. M. Oguni and C. A. Angell, J. Chem. Phys., 1980, 73, 1948-1954.

69. P. Gallo, M. Rovere and E. Spohr, Phys. Rev. Lett., 2000, 85, 4317-4320.

70. N. Nandi, B. Bagchi, J. Phys. Chem. A, 1998, 102, 8217-8221.

71. S. K. Pal, J. Peon, B. Bagchi and A. H. Zewail, J. Phys. Chem. B, 2002, 106, 1237612395.

72. W. Gotze, Complex Dynamics of glass-forming liquids: a mode-coupling theory, 2009, Oxford University Press, Oxford.

73. W. Gotze, J. Phys.: Condens. Matter, 1999, 11, A1-A45.

74. W. Gotze and L. Sjogren, Rep. Prog. Phys., 1992, 55, 241-376.

75. A. Angell, Nature, 1998, 393, 521-524.

76. W. Gotze and L. Sjogren, Chem. Phys., 1996, 212, 47-59.

77. C. Masciovecchio, S. C. Santucci, A. Gessini, S. Di Fonzo, G. Ruocco and F. Sette, Phys. Rev. Lett., 2004, 92, 255507.

78. P. Gallo, F. Sciortino, P. Tartaglia and S. H. Chen, Phys. Rev. Lett., 1996, 76, 2730-2733.

79. R. C. Welch, J. R. Smith, M. Potuzak, X. Guo, B F. Bowden, T. J. Kiczenski, D. C. Allan, E. A. King, A. J. Ellison and J. C. Mauro, Phys. Rev. Lett., 2013, 110, 265901.

80. M. Potuzak, R. C. Welch and J. C. Mauro, J. Chem. Phys., 2011, 135, 214502.

81. L. Liang, P. Rulis, L. Ouyang and W. Y. Ching, Phys. Rev. B, 2011, 83, 024201.

82. N. Giovambattista, F. W. Starr, F. Sciortino, V. Buldyrev and H. E. Stanley, Phys. Rev. E, 2002, 65, 041502.

83. F. Sciortino, A. Geiger and H. E. Stanley, Nature, 1991, 354, 218-221.

84. D. Laage and J. T. Hynes, Science, 2006, 311, 832-835.

85. D. Laage and J. T. Hynes, Chem. Phys. Lett., 2006, 433, 80-85.

86. D. Laage and W. H. Thompson, J. Chem. Phys., 2012, 136, 044513.

87. D. Laage, G. Sirnemann, F. Sterpone and J. T. Hynes, Acc. Chem. Res., 2012, 45, 53-62.

88. A. Geiger, M. Kleene, D. Paschek, A. Rehtanz, J. Mol. Liq., 2003, 106/2-

3, 131-146.

89. G. Sutmann and R. Vallauri, J. Mol. Liq., 2002, 98-99, 213-224. 


\section{TABLES}

Table 1. Experimental $\left(\mathrm{D}_{\mathrm{exp}}\right)$ and calculated $\left(\mathrm{D}_{\text {calc }}\right)$ self-diffusion constants for liquid water at ambient and low temperature. The temperature dependent parameter taut is the coupling constant time in the damping factor $\gamma=$ $m /$ taut of the Langevin equation of motion $m\left(\mathrm{~d}^{2} \mathrm{r} / \mathrm{dt}^{2}\right)=\mathrm{F}-\gamma(\mathrm{dr} / \mathrm{dt})+\eta(\mathrm{t})$, where $\eta(\mathrm{t})$ is a noise term while $m, \mathrm{r}$ and $\mathrm{F}$ are, respectively, the mass, the position and the force field acting on the atom under consideration in the dynamics (see Section 2 for details). ${ }^{46-47}$

$\mathrm{T}(\mathrm{K}) \quad \mathrm{D}_{\exp } \times 10^{5}\left(\mathrm{~cm}^{2} / \mathrm{s}\right) \quad \mathrm{D}_{\text {calc }} \times 10^{5}\left(\mathrm{~cm}^{2} / \mathrm{s}\right) \quad$ taut $(\mathrm{ps})$

\begin{tabular}{llll}
\hline & & & \\
$300 \pm 1$ & $2.40^{\mathrm{a}}$ & $2.38 \pm 0.03$ & $0.210 \pm 0.002$ \\
$288 \pm 1$ & $1.77^{\mathrm{a}}$ & $1.78 \pm 0.01$ & $0.154 \pm 0.002$ \\
$273 \pm 1$ & $1.10^{\mathrm{a}}$ & $1.08 \pm 0.02$ & $0.100 \pm 0.002$ \\
$258 \pm 1$ & $0.53^{\mathrm{b}}$ & $0.516 \pm 0.007$ & $0.052 \pm 0.002$ \\
$248 \pm 1$ & $0.31^{\mathrm{b}}$ & $0.308 \pm 0.007$ & $0.036 \pm 0.002$ \\
$243 \pm 1$ & $0.20^{\mathrm{b}}$ & $0.199 \pm 0.004$ & $0.025 \pm 0.002$ \\
\hline
\end{tabular}

a) M. Holz, S. R. Heil and A. Sacco, Phys. Chem. Chem. Phys., 2000, 2, 4740-4742

b) K. T. Gillen, D. C. Douglass and M. J. R. Hoch, J. Chem Phys., 1972, 57, 5117-5119

Table 2. Ambient and low temperature stretched exponential parameters for the relaxation of residence times in a nanodrop of liquid water modelled as a sphere of nanometric dimensions. See Sections 3.1 and 3.2 for details.

\begin{tabular}{lrl}
\hline $\mathrm{T}(\mathrm{K})$ & \multicolumn{1}{c}{$\tau(\mathrm{ps})$} & $\beta$ \\
\hline $300 \pm 1$ & $75.3 \pm 1$ & $0.58 \pm 0.01$ \\
$288 \pm 1$ & $101.3 \pm 1$ & $0.60 \pm 0.01$ \\
$273 \pm 1$ & $163.6 \pm 1$ & $0.59 \pm 0.01$ \\
$258 \pm 1$ & $334 \pm 3$ & $0.58 \pm 0.01$ \\
$248 \pm 1$ & $542 \pm 5$ & $0.60 \pm 0.01$ \\
$243 \pm 1$ & $998 \pm 9$ & $0.59 \pm 0.01$ \\
\hline
\end{tabular}




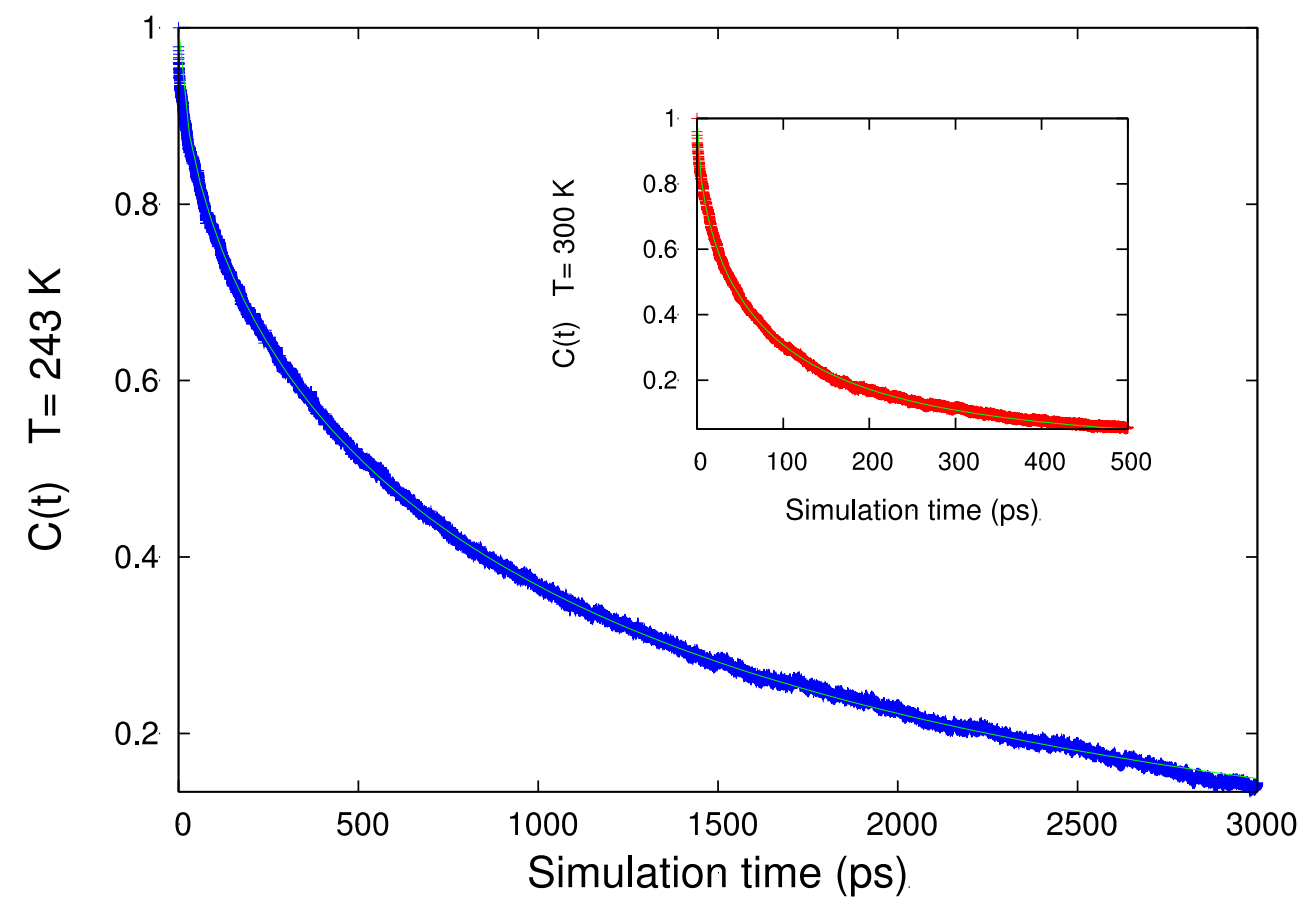

Fig. 1. Normalized residence time correlation functions for liquid water. $\mathrm{C}(\mathrm{t})$ represents the number of water molecules that remain in the region of interest at a given time $t$ with respect to that number at the initial time. In this case the region of interest considered is a nanodrop of bulk water at $300 \mathrm{~K}$ (inset above) and $243 \mathrm{~K}$ (curve below). The symbols are data points obtained from molecular dynamics simulations performed on neat water using eqn (1) (see Section 3.1 and 3.2 for details). The continuous line represents the best fit using a stretched exponential function $\exp [$ $-(\mathrm{t} / \tau)^{\beta}$ ] (see Table 2 for results). 


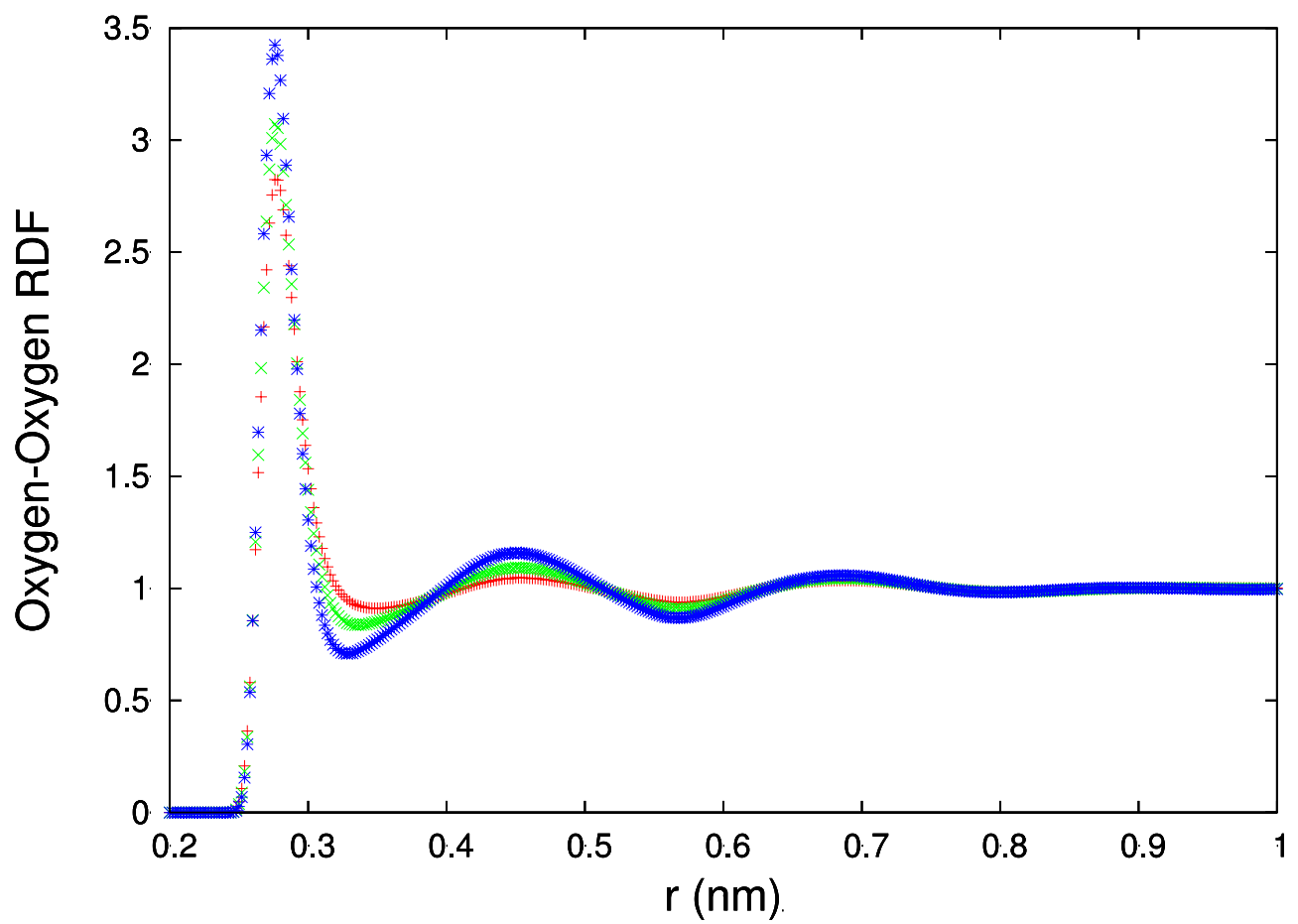

Fig. 2. Oxygen-Oxygen radial distribution functions (goo) for bulk water at $300 \mathrm{~K}$ (+: red), $273 \mathrm{~K}$ (x: green) and $243 \mathrm{~K}(*$ : blue) showing the liquid character of the system at $\mathrm{T} \leq 273 \mathrm{~K}$. Data calculated using the GROMACS software package. 


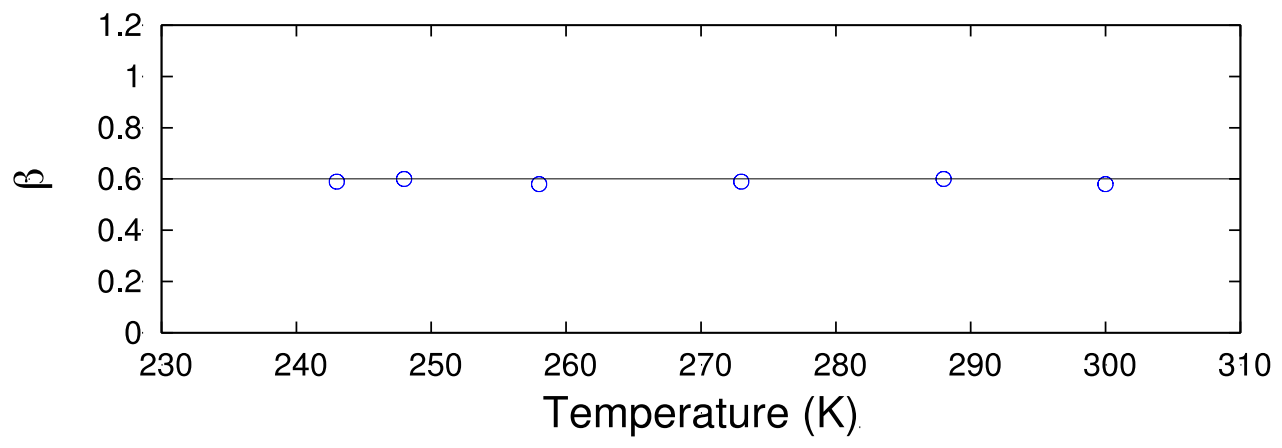

Fig. 3. The temperature independence of the stretched exponent $\beta$ is shown for a nanodrop of liquid water, modelled as a sphere of nanometric dimensions, cooled from 300 to $243 \mathrm{~K}$. See Fig. 1 and Section 3.2 for details. 


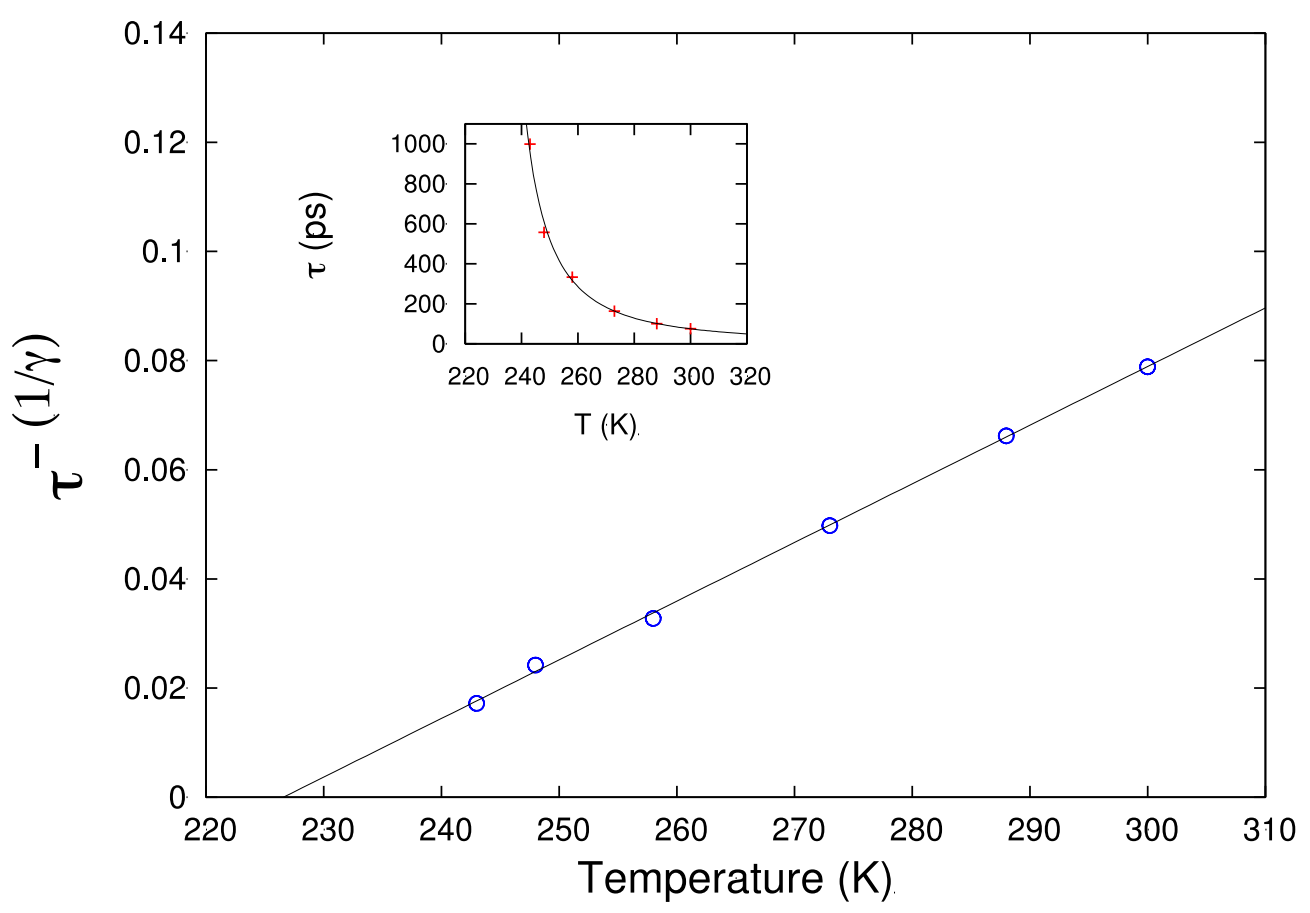

Fig. 4. Decay dynamics for liquid water from $300 \mathrm{~K}$ down to $243 \mathrm{~K}$. The magnitude $\tau$ is the effective relaxation time obtained from the fit of the molecular dynamics simulations data to a stretched exponential function $\exp \left[-(\mathrm{t} / \tau)^{\beta}\right]$ (see Fig. 1 and Section 3.2 for details). The straight line illustrates the power-law divergence $\tau \sim\left(\mathrm{T}-\mathrm{T}_{\mathrm{S}}\right)^{-\gamma}$ with best fit for $\mathrm{T}_{\mathrm{S}}=227 \pm 3 \mathrm{~K}$ and $\gamma=1.71 \pm$ 0.10 . 


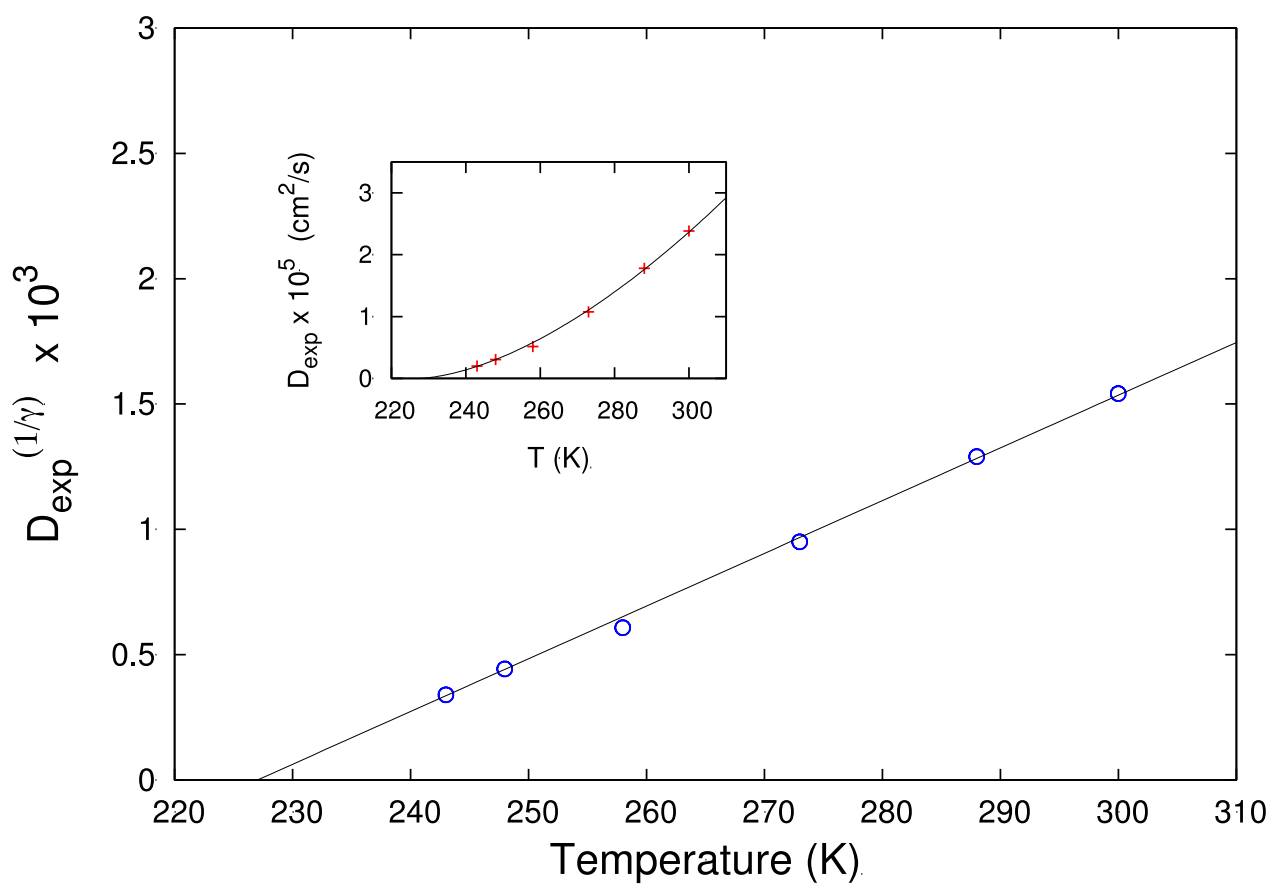

Fig. 5. Evolution of the experimental diffusion coefficient, $D_{\text {exp }}$, for liquid water in the temperature regime $300-243 \mathrm{~K}$ (see Table 1 ). The straight line illustrates the power-law vanishing $D_{\square} \sim\left(\mathrm{T}-\mathrm{T}_{\mathrm{S}}\right)^{\gamma}$ with best fit for $\mathrm{T}_{\mathrm{S}}=227 \pm 1 \mathrm{~K}$ and $\gamma=1.64 \pm 0.10$ (see Section 3.2). 


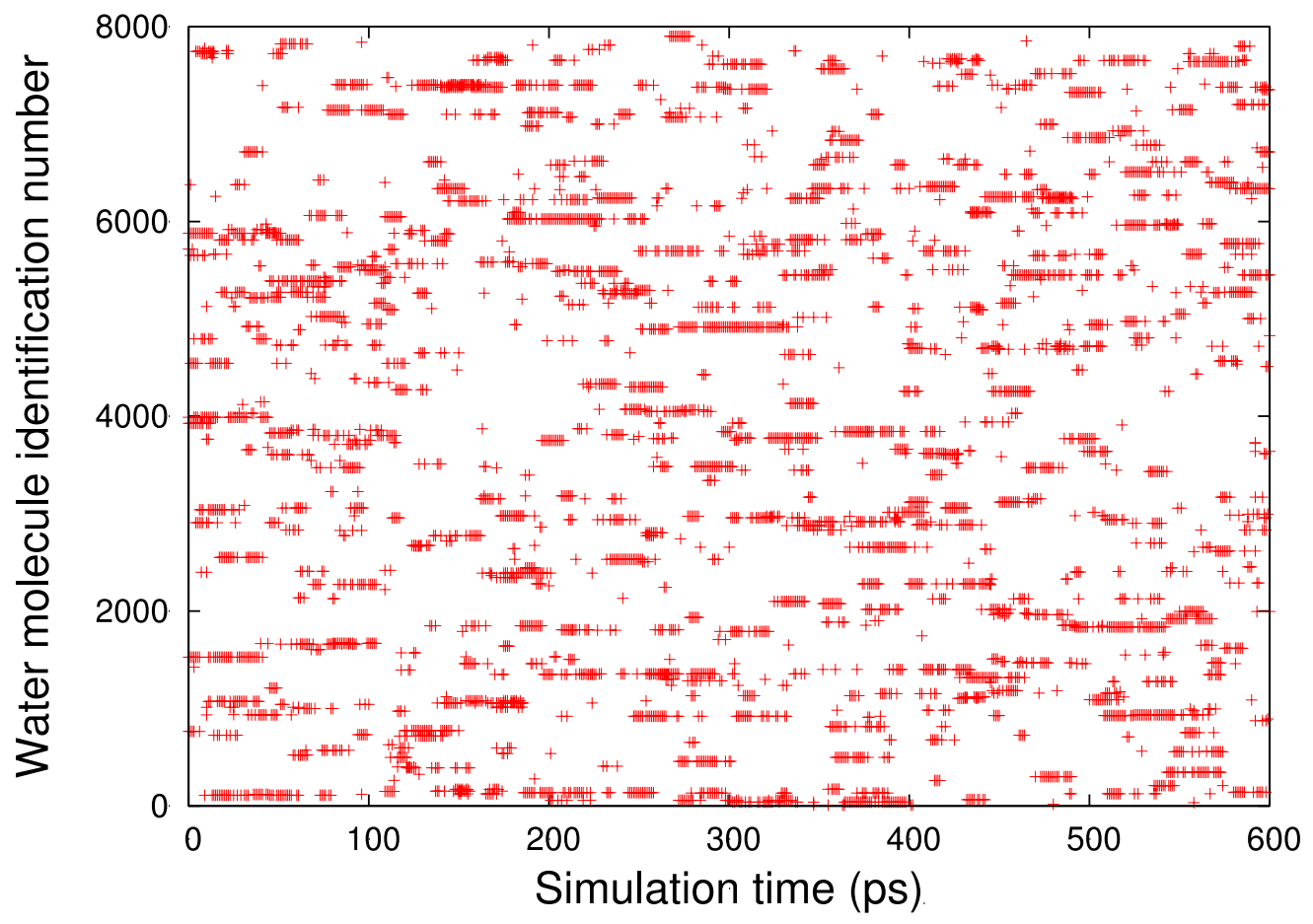

Fig. 6. Map of residence times for water molecules located at a distance smaller than a cutoff of $0.40 \mathrm{~nm}$ from a given solvent molecule taken as a reference ( $\mathrm{rt}(\mathrm{r}=0.40 \mathrm{~nm})$ ). The data corresponds to room temperature bulk water at $\mathrm{T}=300 \mathrm{~K}$. If a given water molecule ( $y$-coordinate), at a particular time of the simulation ( $x$-coordinate), is located at a distance of the reference smaller than the cutoff, then a cross appears at the corresponding $(x, y)$ location. If the distance is higher or equal than the cutoff, a blank is settled instead. The length of the lines inside the figure provides the value of the resident time $\mathrm{rt}(\mathrm{r}=0.40 \mathrm{~nm})$ for each water molecule. In some cases the lines reduce to a single point. Temporal resolution: $0.1 \mathrm{ps}$. The presence of multiple time scales can be clearly seen at this atomic level. Please mind the $x$-axis scaling when comparing with low temperature data shown in Fig 8. Data calculated using the GROMACS software package. 

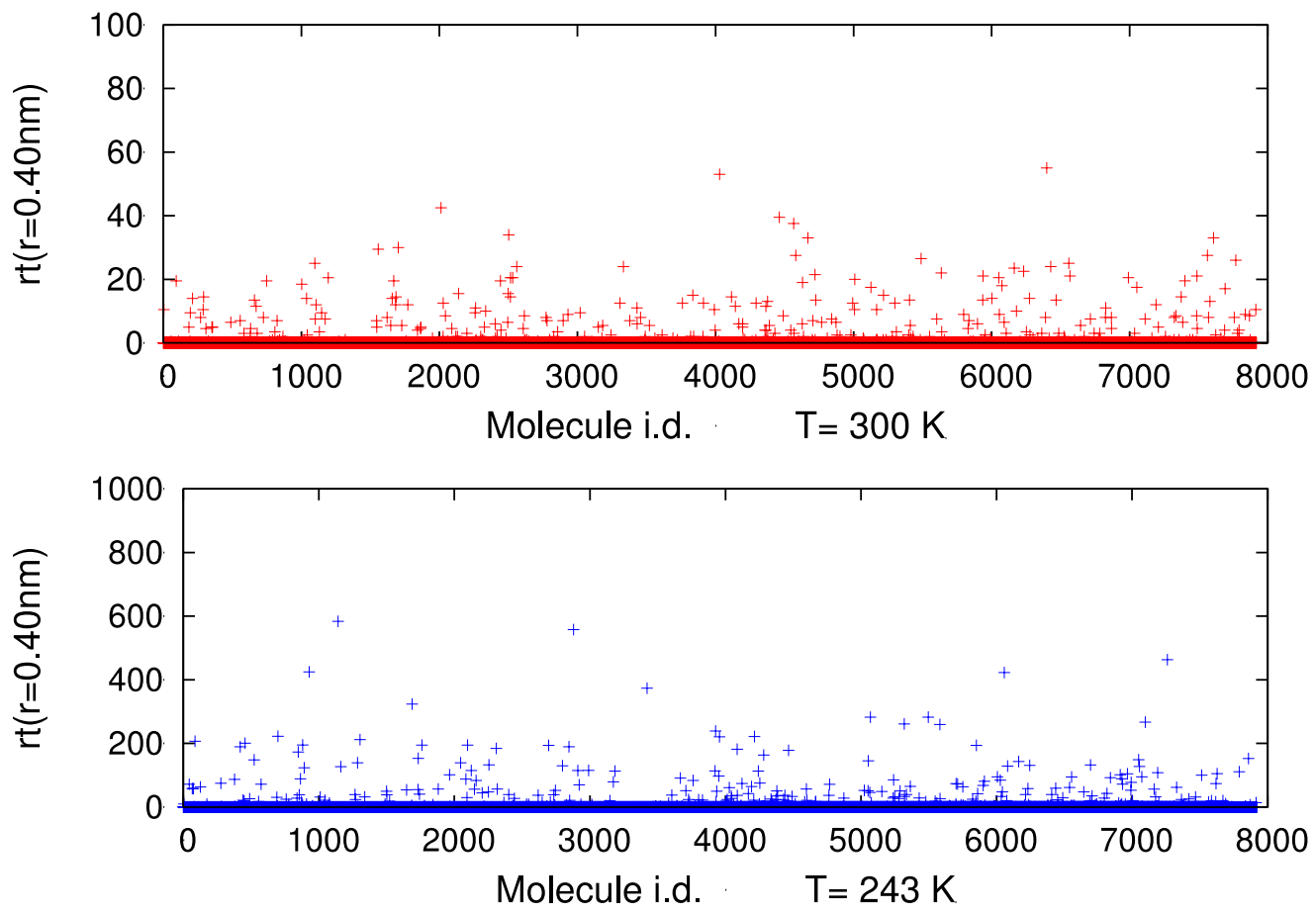

Fig. 7. Residence times of water molecules located at a distance smaller than a cutoff of $0.40 \mathrm{~nm}$ from a given solvent molecule taken as a reference $(\mathrm{rt}(\mathrm{r}=0.40 \mathrm{~nm}))$. Time units: picoseconds. The maximum value of those residence times increases one order of magnitude when water is cooled from $300 \mathrm{~K}$ (upper part of the figure) down to $243 \mathrm{~K}$ (lower part). Please mind the $y$-axis scaling in both cases (upper and lower representations). Data calculated using the GROMACS software package. 


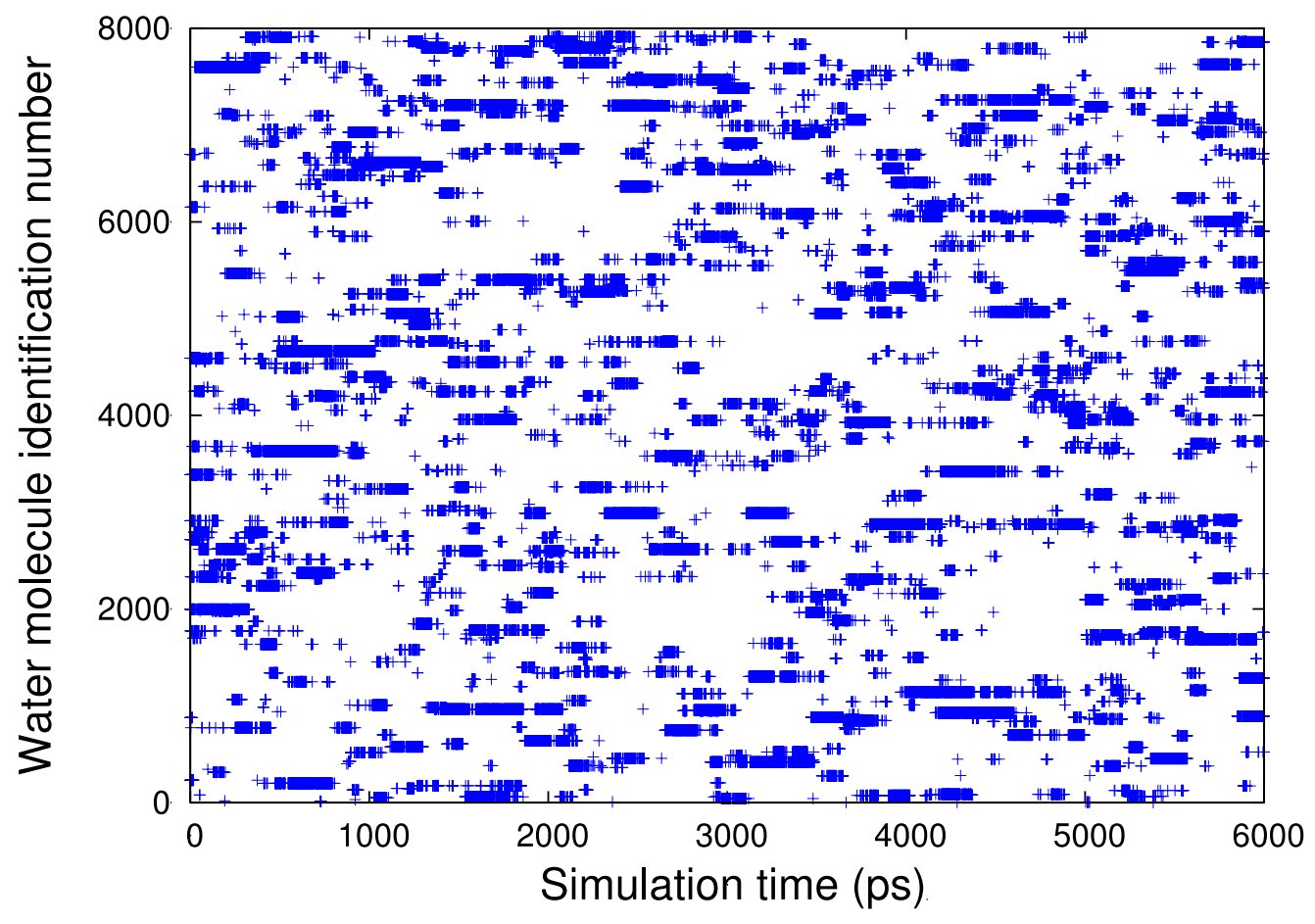

Fig. 8. Map of residence times for water molecules located at a distance smaller than a cutoff of $0.40 \mathrm{~nm}$ from a given solvent molecule taken as a reference $(\mathrm{rt}(\mathrm{r}=0.40 \mathrm{~nm})$ ). The data corresponds to low temperature bulk liquid water at $\mathrm{T}=243 \mathrm{~K}$. If a given water molecule ( $y$-coordinate), at a particular time of the simulation ( $x$-coordinate), is located at a distance of the reference smaller than the cutoff, then a cross appears at the corresponding $(x, y)$ location. If the distance is higher or equal than the cutoff, a blank is settled instead. The length of the lines inside the figure provides the value of the resident time $\mathrm{rt}(\mathrm{r}=0.40 \mathrm{~nm})$ for each water molecule. In some cases the lines reduce to a single point. Temporal resolution: $0.1 \mathrm{ps}$. The presence of multiple time scales can be clearly seen at this atomic level. Please mind the $x$-axis scaling when comparing with room temperature data shown in Fig 6. Data calculated using the GROMACS software package. 


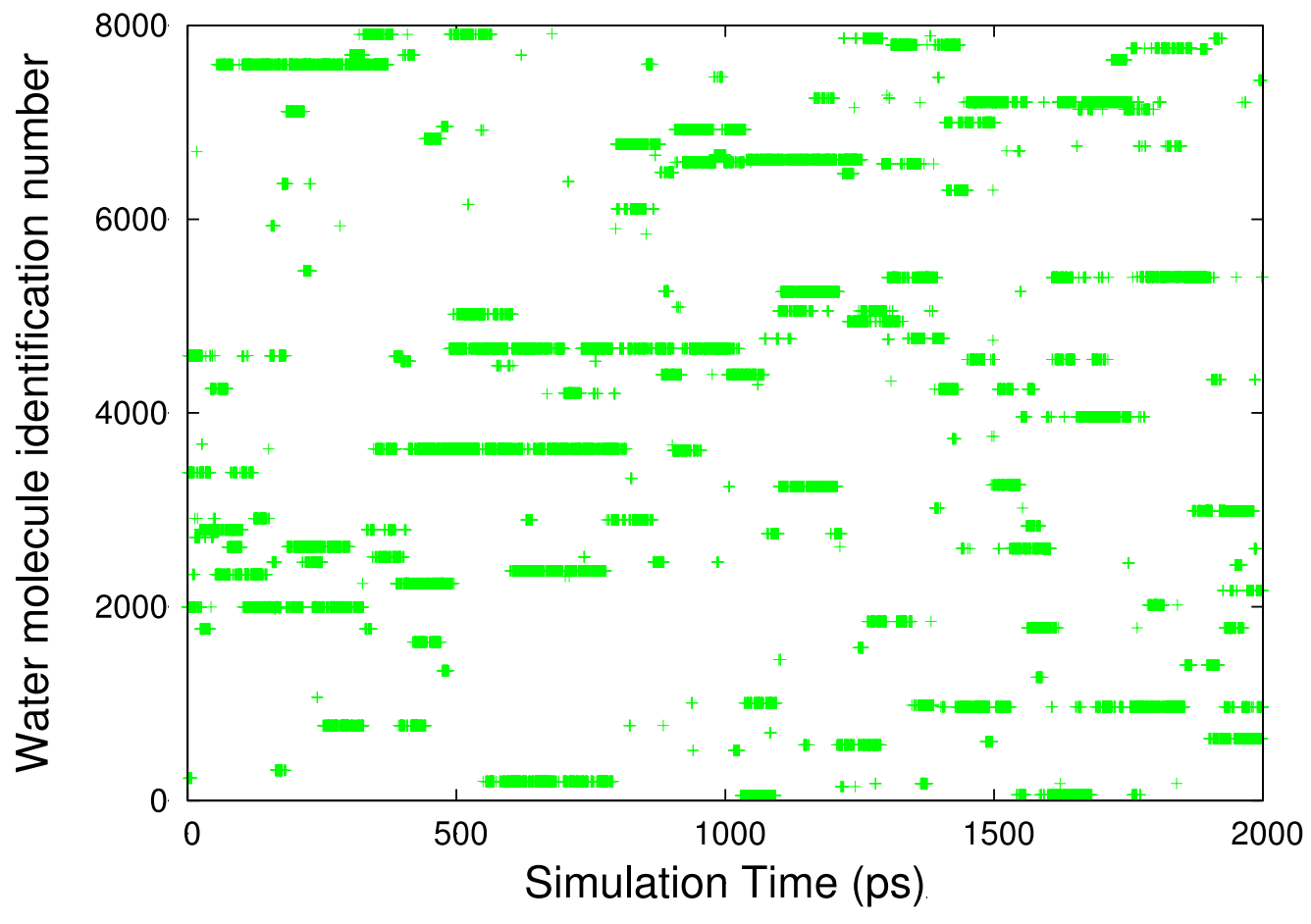

Fig. 9. Map of residence times for water molecules located at the smallest distance of a given solvent molecule taken as a reference. If a given water molecule ( $y$-coordinate), at a particular time of the simulation ( $x$-coordinate), is located at the smallest distance of the solvent molecule of reference, then a cross appears at the corresponding $(x, y)$ location. On the contrary if it is not situated at that smallest distance, a blank is settled instead. The length of the lines inside the figure provides the value of the resident time for each water molecule. In some cases the lines reduce to a single point. Temporal resolution: $0.1 \mathrm{ps}$. This figure shows that, at a given time, success in being located at the smallest distance of the reference does not necessarily imply success in staying at such a minimum distance during a long period of time. Data calculated on bulk liquid water at $\mathrm{T}=243 \mathrm{~K}$ using the GROMACS software package. 

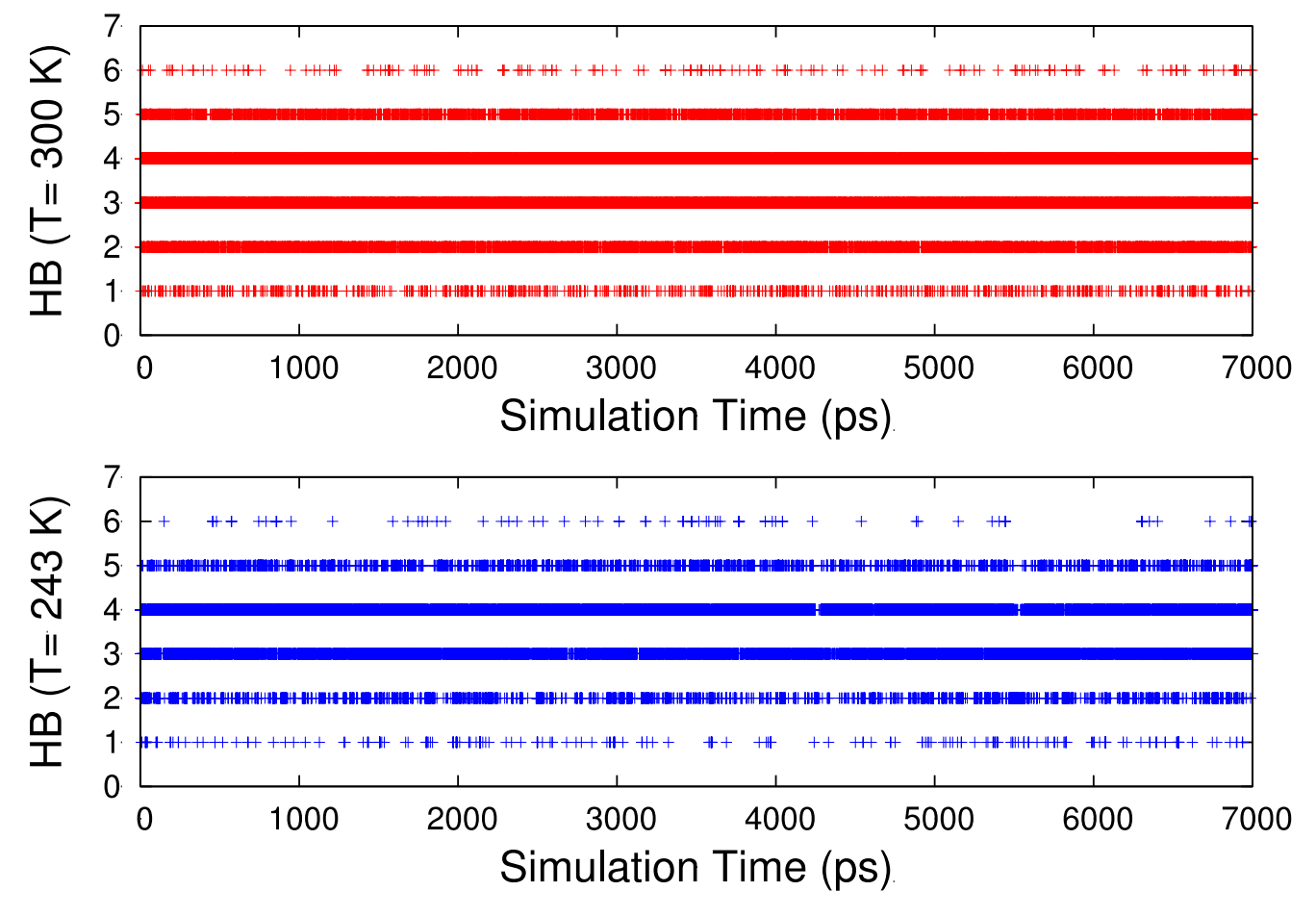

Fig. 10. Number of hydrogen bonds (HB) that a given water molecule taken as a reference can establish with their neighbours at room and low temperature (300 and $243 \mathrm{~K}$, respectively). As shown, the coordination of the first hydration shell as a function of time presents up to six hydrogen bonds per water molecule. Moreover, the number of 'defects' decreases with temperature: a more perfect tetrahedral hydrogen bond network is approached for low temperature data (list of percentages of hydrogen bonds at $300 \mathrm{~K}$ and $243 \mathrm{~K}$, respectively: $1.3 \%$ and $0.3 \%$ (1 $\mathrm{HB}$ ), $10.7 \%$ and $5.2 \%$ (2 HB), $35.0 \%$ and $27.6 \%$ (3 HB), $45.5 \%$ and $59.8 \%$ (4 HB), $7.3 \%$ and $7.0 \%$ (5 HB), $0.2 \%$ and $0.1 \%$ (6 HB) ). Time resolution: 0.1 ps. See text for more information (Section 4.3). Data calculated using the GROMACS software package. 
A method to predict magnitudes in quantitative agreement with experimental data has been devised and applied to model water-water interactions at low temperature.

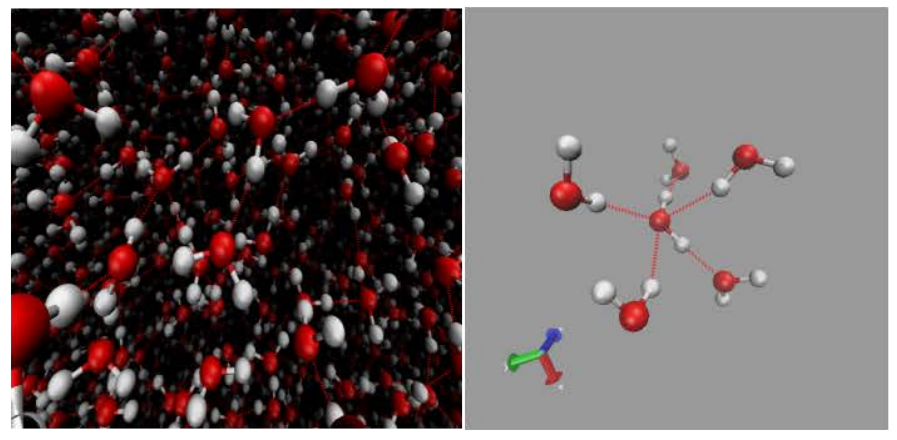

